\title{
Research on Synthesis and Properties of Polyaniline
}

Le Luo

Chongqing Vocational Institute of Engineering, Chongqing, China, 402260

\begin{abstract}
Polyaniline is widely used in many fields due to its many advantages such as easy access to raw materials, simple synthesis process, good chemical properties and environmental stability. In this paper, the synthesis methods of conducting polymer polyaniline are discussed firstly. Then, the conductivity, particle size and infrared spectrum are discussed to provide some references for the relative researchers.
\end{abstract}

Keywords: polyaniline, synthesis, polymerization

\section{Introduction}

Polyaniline is a kind of high molecular compound with special electrical and optical properties, which is electrically conductive and electrochemical. After a certain treatment, it can make various kinds of equipment and materials with special functions, such as biological or chemical sensors, electronic field emission source urease sensor, compared with the traditional lithium electrode material in the process of charge and discharge has more excellent reversibility of electrode materials, selective membrane materials, antistatic and electromagnetic shielding materials, anti-corrosion materials, etc.. Polyaniline has been widely studied and applied because of its advantages such as easy access to raw materials, simple synthesis process and good chemical and environmental stability. The electric activity source of Polyaniline in the molecular chain of $\mathrm{P}$ electron conjugated structure. The expansion of $\mathrm{P}$ electronic system is in the molecular chain. The $\mathrm{P}$ bonding state and $\mathrm{P}^{*}$ antibonding states respectively form the valence band and conduction band. The non $\mathrm{P}$ electron conjugated structure localized by doping can form the $\mathrm{P}$ type and $\mathrm{N}$ type conducting state. The doping mechanism of different cation vacancies in produce oxidation agents from other 
conductive polymers, the number of electrons in polyaniline doping process does not change, but by the proton acid doped into the backbone of decomposition and anion, combined with amine and imine groups in atom dipole and dipole delocalization to the key of the molecular chain, so that the conductive polyaniline showed a higher. This unique doping mechanism makes the doping and de doping of polyaniline completely reversible, and the doping degree is affected by the value and the electric potential, and the corresponding change of the appearance colour. Polyaniline has electrochemical and electrochromic properties. In this paper, the chemical synthesis method was used to synthesize polyaniline and its properties were studied.

\section{Synthesis of Polyaniline}

The chemical synthesis of polyaniline is the oxidation polymerization of aniline monomer in the acidic medium. It is the most common method to prepare polyaniline by chemical method. Using HCI as medium with 2S208 as oxidant, synthesis of $22500 \mathrm{~g}$ polyaniline for one-time to use aniline chemical synthesis of polyaniline is mainly affected by the reaction medium acid type and concentration of oxidant type and concentration of monomer concentration and reaction temperature, reaction time and other factors. The electrochemical polymerization of polyaniline mainly includes: constant current method, scanning method and pulse polarization method. Generally, aniline is polymerized on the anode in an acidic solution. Electrode material, electrode potential, electrolyte solution $\mathrm{pH}$ value and the type of polymerization of aniline have certain effect of operation process, which are as follows: ammonia and hydrofluoric acid was prepared by the reaction of electrolyte solution, the platinum wire as the counter electrode and platinum electrode as working electrode, $\mathrm{Cu} / \mathrm{CuF}$ as reference electrode in an electrolytic cell containing electrolyte. The electrochemical polymerization was carried out by dynamic potential scanning $(\mathrm{E}=0.6 \mathrm{~V} \sim 2.0 \mathrm{~V})$. After a period of reaction, polyaniline was firmly adsorbed on the electrode.

In this paper, the synthesis method of polyaniline is the chemical method. In an electric mixer $500 \mathrm{~mL}$ three mouth flask, add $200 \mathrm{~mL}$ distilled water, then adding a certain amount of twelve benzene sulfonic acid and hydrochloric acid, stirring until completely dissolved, adding n-butanol as surfactant. We take a certain amount of aniline, stirring dripping, with aniline in emulsion, quickly become white. Continue to stir $0.5 \mathrm{H}$. Adjust the temperature to $0 \sim 5 \mathrm{Deg}$. C, weigh a certain amount of ammonium persulfate dissolved in distilled water to prepare solution of $1 \mathrm{~mol}$ and L-1, slowly dropped into the three mouth flask, $0.5 \mathrm{~h}$ infusion: with the addition of oxidant ammonium persulfate, emulsion from white to light blue, turned into dark green. Continue to maintain the temperature response of $6 \mathrm{~h}$, adding acetone emulsification, end reaction: filtration, get a dark green solid. With anhydrous ethanol and deionized water washed until the filtrate is colorless, the solid in 60 Deg. C. The hydrochloric acid molar ratio, acid dosage, polymerization time, polymerization temperature, the effects of synthesis conditions on the conductivity of polyaniline. The microstructure analysis of doped polyaniline resolution using transform infrared spectrometer for the $4 \mathrm{~cm}-1$ 
sampling rate of $80 \mathrm{~S}^{-1}$ scanning frequency range $400-4000 \mathrm{~cm}^{-1}$; using thermogravimetric analyzer on doped polyaniline thermal stability, the heating rate of 20 DEG.Min-1, nitrogen gas atmosphere; conductive polyaniline solid powder into ID10 Abrasives after drying. The tablet machine made of tablet diameter is $10 \mathrm{~mm}$ the thickness of not more than $4 \mathrm{~mm}$. The conductivity was measured by four probe tester of doped polyaniline.

\section{Properties of Polyaniline}

\subsection{Electronic conductivity analysis}

The concentration of methyl sulfonic acid is a variable. The other experimental conditions remain unchanged aniline volume fraction of $5 \%$, oxidant and monomer molar ratio of $1: 1$. The electronic conductivities are shown in Table1.

Table 1 Effects on electronic conductivity of concentration

\begin{tabular}{|c|c|}
\hline Acid Concentration $(\mathrm{mol} / \mathrm{L})$ & Electronic Conductivity $(\mathrm{S} / \mathrm{cm})$ \\
\hline 1 & 2.93 \\
\hline 1.5 & 5.38 \\
\hline 2 & 4.39 \\
\hline 2.5 & 3.86 \\
\hline 3 & 2.19 \\
\hline
\end{tabular}

When the concentration of methyl acid was changed, the overall trend of conductivity increased first and then decreased. In this part of the experiment, methyl sulfonic acid was used as a dopant and an acid environment provider. When the acid is strong, the conductivity of the polymer is high; the organic acid concentration is too low will have a negative contribution to conductivity. When the sulfonic acid concentration is too low, the acidity of the system is insufficient, and the conductivity of the product is low. When the sulfonic acid concentration is too high, the excess organic sulfonic acid in the product will block the electron transfer between the molecular chains, and reduce the conductivity of the product. In the former part of the experiment, the conductivity of polyaniline doped with hydrochloric acid is $5.38 \mathrm{~S} / \mathrm{cm}$, and the conductivity of the product is higher. With the increase of aniline dosage, the hydrochloric acid was consumed gradually, which led to the increase of the system and the inhibition of the reaction process. Therefore, in theory, the electrical conductivity should be gradually increased and maintained at a certain level. However, the linear increase of the actual conductivity may be due to the increase of aniline dosage, which is beneficial to the formation of large molecular weight polyaniline products. Because the conduction velocity is much larger than the inter chain conduction velocity, the conductivity increases when the molecular weight increases gradually. And in the case of aniline excess, the hydrochloric acid in the system is fully consumed, and the doping degree of the product is high. However, do not rule out the possibility 
of product in water absorption and compression process leads to large errors in the conductance measurement method of four.

\subsection{Particle size analysis}

The laser particle size analyzer is to use laser as the light source, to disperse the particles in the medium for diffraction and scattering, the data collection, the final size distribution. When the laser is used as the light source, the spatial distribution of the light energy is only related to the particle size. For the diffraction of the particles, the size of each particle determines the size of the light energy corresponding to each specific angle. The proportion of specific light energy in the total light energy should reflect the distribution abundance of each particle. First, we use a glass rod to stir, so that the sample evenly dispersed, and then disperse three minutes ultrasound. Under normal circumstances, the use of ethanol dispersion, this experiment due to more samples, so the use of deionized water to disperse. It is important to note that the amount of sample must be eliminated in the process of dispersion. During the test, the sample through a circulation pump evenly is distributed in the whole system, multiple measurements until the relatively small change in shape. The size of the particles depends on the dispersion and is easy to agglomerate. Therefore, the data of particle size analysis is only used for qualitative analysis. The average particle size of different mixed oxidant molar ratio showed that the average particle size of the product decreased first and then increased. To separate oxidizer particle size distribution of ammonium sulfate oxidation reaction rate in the process of strong, fast, prone to local overheating, the occurrence of violent, products of the larger particle size; iron oxide as the oxidant was two level continuous distribution. In theory, with the increase of the ratio of ammonium persulfate, the average particle size should be increased. There are only one set of outliers, and the particle size is nearly one order of magnitude higher than that of the other groups. Therefore, the data of the comparison group is likely to be the experimental error, so the exclusion of the remaining five groups of data in comparison with the theoretical inference. The particle size increased with the increase of ammonium persulfate ratio. The different average particle sizes of different mole ratios are shown in Figure 1. 


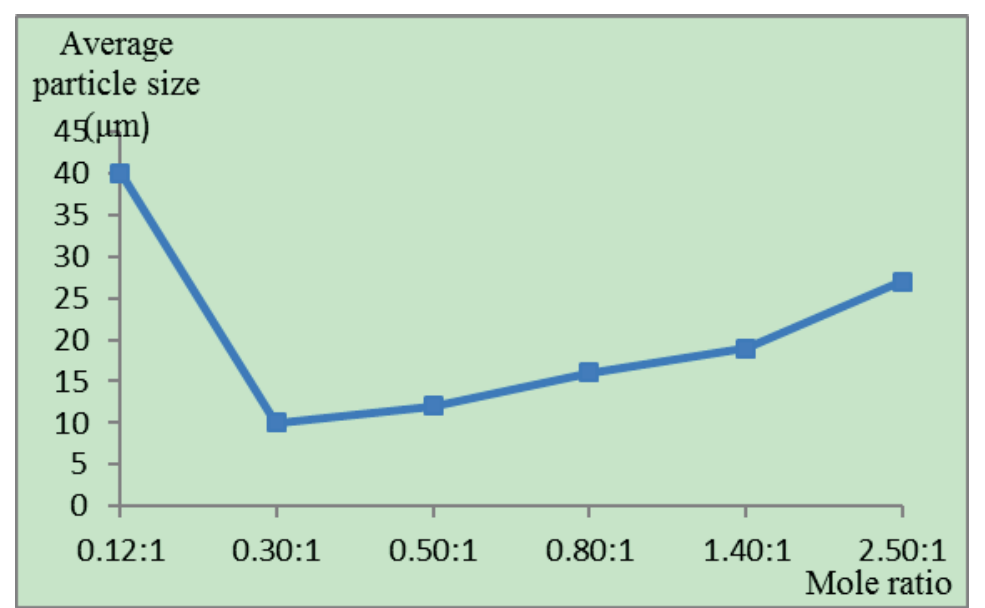

Fig. 1: Different average particle sizes of different mole ratios

\subsection{Infrared spectrum analysis}

In organic molecules, the atoms that form chemical bonds or functional groups are in a state of constant vibration. So, using infrared irradiation of organic molecules, molecules in chemical bonds or groups can produce shock absorption, different chemical bonds or functional groups in different absorption frequency, infrared spectrum, is in a different position so as to contain what chemical bonds or functional groups in the molecule to obtain information. To measure the different molecular bond sample molecules produced in different vibration frequency, and the absorption of infrared spectra of these molecules can bond through the detector acceptance, results were processed to record relevant data through Fourier transform function, the instrument analysis. In order to prepare the sample, the powder and powder were mixed in a certain proportion, and then pressed into thin slices. It is worth noting that the sample process, need to mold a thorough cleaning with alcohol, and heating and drying with sodium tablet attention when powder evenly, the sheet thickness. The vibrational modes of molecules can be divided into two categories: stretching and bending vibrations. The former refers to the reciprocating motion of the atoms along the axis of the bond. The latter refers to the vibration of the atoms perpendicular to the chemical bond, which is usually represented by different symbols. However, it does not appear on a fixed wave number, which is the specific wave number, which is related to the environment of the group in the molecule, which leads to the frequency shift of the group. After all the doped polyaniline absorption peak will transfer to the low frequency direction in different degree, this is due to the doping density of electron cloud in the polymer chain decreased, induced effect and total effect of the car. The diagram of the infrared spectrum analysis is shown in Figure 2. 


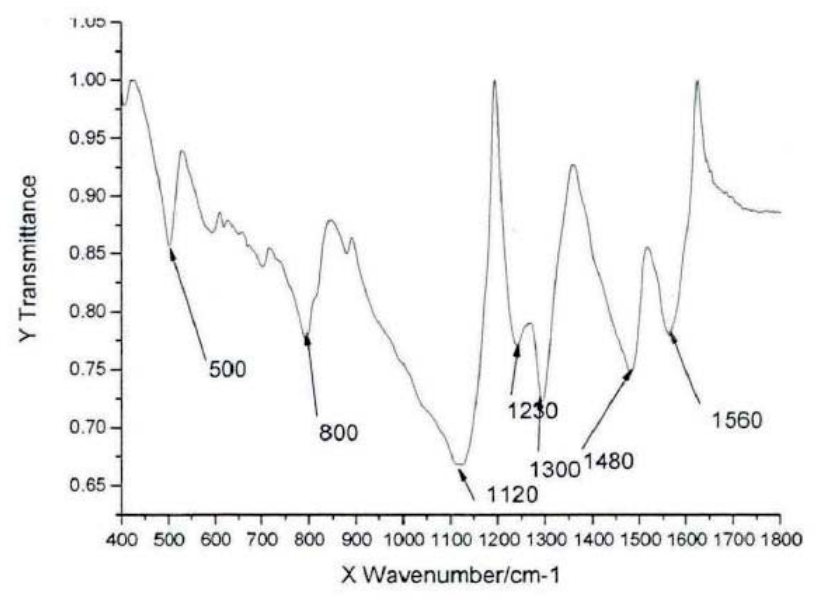

Fig. 2: Diagram of the infrared spectrum analysis

\section{Conclusion}

The diversity and the superiority of the polyaniline synthesis method determine its wide application prospect. With the unremitting efforts of many scientists, the research on the structure, properties, synthesis, doping, modification, and other aspects of the polyaniline has made considerable progress. Now, people are strengthening the research intensity of polyaniline. We believe that the polyaniline can be more widely used due to its superior properties.

\section{References}

[1] Zheng Shiguo, Zhan Haoqiang, Synthesis and Research on Properties of a Novel Quaternary Ammonium Polyaniline Copolymers, 21(4), pp. 424-427, 2013. [2] Luo Yunqing, Dong Yao, Ning Bo, Zhang Xia, Ding Tianwang, Zhang Qun, Liu Wencong, Study on synthesis and performance application of polyaniline nanotubes, Journal of Northeast Normal University (Natural Science Edition), 46(4), pp. 90-94, 2014

[3] Hu Hongchao, Shu Xuguang, Cui Yingde, Progress in synthesis and mechanism of polyaniline, 35(S1), Chemical Industry and Engineering Progress, 35(1), pp. 195-201, 2016.

[4] Wang Chao, New synthetic process for the preparation of PANi with high conductivity, Technique \& Education, 29(2), pp. 27-29+26, 2014.

[5] Liu Dong, Wu Zhenyu, Chang Xiaowei, Li Yanyan, Ding Yigang, Study of the Electrochemical Synthesis Conditions on Anti-corrosion Performance of Polyaniline Coating, Journal of South-Central University for Nationalities (Natural Science Edition), 35(1), pp. 23-28, 2016. 